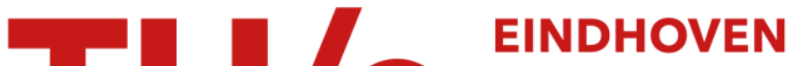

\section{Multimode equivalent networks for the design and analysis of frequency selective surfaces}

\section{Citation for published version (APA):}

Monni, S., Gerini, G., Neto, A., \& Tijhuis, A. G. (2007). Multimode equivalent networks for the design and analysis of frequency selective surfaces. IEEE Transactions on Antennas and Propagation, 55(10), 2824-2835. https://doi.org/10.1109/TAP.2007.905846

DOI:

10.1109/TAP.2007.905846

Document status and date:

Published: 01/01/2007

\section{Document Version:}

Publisher's PDF, also known as Version of Record (includes final page, issue and volume numbers)

\section{Please check the document version of this publication:}

- A submitted manuscript is the version of the article upon submission and before peer-review. There can be important differences between the submitted version and the official published version of record. People interested in the research are advised to contact the author for the final version of the publication, or visit the $\mathrm{DOI}$ to the publisher's website.

- The final author version and the galley proof are versions of the publication after peer review.

- The final published version features the final layout of the paper including the volume, issue and page numbers.

Link to publication

\section{General rights}

Copyright and moral rights for the publications made accessible in the public portal are retained by the authors and/or other copyright owners and it is a condition of accessing publications that users recognise and abide by the legal requirements associated with these rights.

- Users may download and print one copy of any publication from the public portal for the purpose of private study or research.

- You may not further distribute the material or use it for any profit-making activity or commercial gain

- You may freely distribute the URL identifying the publication in the public portal.

If the publication is distributed under the terms of Article 25fa of the Dutch Copyright Act, indicated by the "Taverne" license above, please follow below link for the End User Agreement:

www.tue.nl/taverne

Take down policy

If you believe that this document breaches copyright please contact us at:

openaccess@tue.nl

providing details and we will investigate your claim. 


\title{
Multimode Equivalent Networks for the Design and Analysis of Frequency Selective Surfaces
}

\author{
Stefania Monni, Member, IEEE, Giampiero Gerini, Member, IEEE, Andrea Neto, Member, IEEE, and \\ Anthon G. Tijhuis, Member, IEEE
}

\begin{abstract}
A modular technique originally proposed for waveguide junctions, the multimode equivalent network approach based on the integral equation formulation (IEMEN), is extended to the analysis of multilayer Frequency Selective Surfaces integrated with waveguide array antennas. This technique represents each layer and transition between layers in terms of a generalized impedance or admittance matrix, obtained directly from the solution of an integral equation with reduced kernel. Thanks to the adopted formulation, the integral equation needs to be solved only in a limited set of frequency points. The IEMEN method is validated by comparison with results available in literature.
\end{abstract}

Index Terms-Admittance matrix, antenna phased arrays, frequency selective surfaces (FSS), impedance matrix, periodic structures.

\section{INTRODUCTION}

$\mathbf{F}$ REQUENCY selective surfaces (FSS) are arrays of periodically arranged metallic patches or apertures on a metallic plane, designed to have a particular filtering behavior as a function of the frequency or/and the angle of incidence. As a typical application, they are used to shape antennas frequency or angular response. Originally, the FSS and the antenna have been studied as stand-alone structures, without taking into account the mutual interactions. The integration of the FSS with the target antenna from the beginning of the design offers interesting possibilities. The end structure is more compact and it can follow the profile of the platform, which is especially important when a low radar cross section (RCS) has to be achieved [1]. Moreover, additional functionalities can be associated to the FSS, as for example realization of improved matching layers and prevention of interference problems [2].

Fig. 1 shows a multilayer FSS, consisting of three planar dielectric layers loaded by patch- and slot-based resonant elements, cascaded to an infinite periodic array of open-ended waveguides. For this kind of structure, either the scattering problem is tackled simultaneously at all layers, by solving the

Manuscript received September 14, 2006; revized April 4, 2007. This work was supported in part by the Netherlands Organisation for Applied Scientific Research (TNO) and in part by the Dutch Ministry of Defence.

S. Monni, G. Gerini, and A. Neto are with Defence, Security and Safety Institute, Netherlands Organization for Applied Scientific Research (TNO), 2597 AK The Hague, The Netherlands (e-mail: stefania.monni@tno.nl; giampiero. gerini@tno.nl; andrea.neto@tno.nl).

A. G. Tijhuis is with the Faculty of Electrical Engineering, Eindhoven University of Technology, 5600 MB Eindhoven, The Netherlands (e-mail: A.G.Tijhuis@tue.nl).

Digital Object Identifier 10.1109/TAP.2007.905846

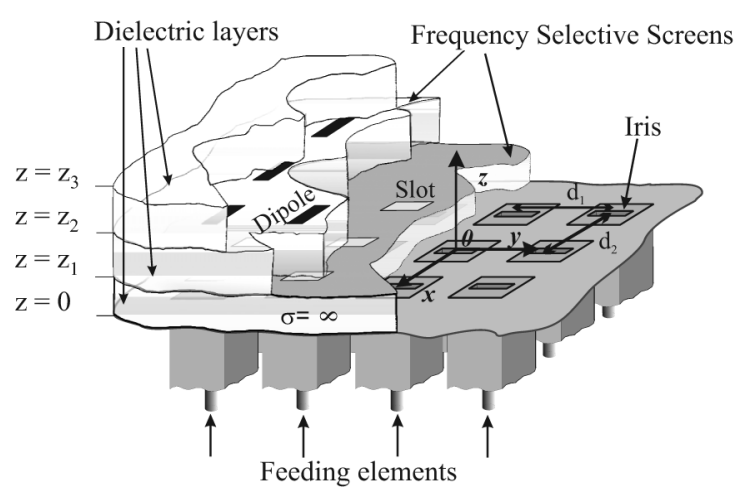

Fig. 1. Geometry of an iris-loaded waveguide array integrated with a generic FSS structure, comprising a slot-based FSS and a patch-based FSS sandwiched between different dielectric layers.

corresponding system of coupled integral equations [3], or it is treated separately at each layer by resorting to a modular methodology.

The latter approach is computationally convenient, as argued in [4]. Moreover, it can be implemented in a general-purpose software tool that allows analyzing an arbitrary number of layers and is therefore an efficient design instrument. Techniques based on the derivation of equivalent microwave networks can be used for this purpose. They were originally developed to study discontinuities in waveguide and waveguide junctions [5], [6] and subsequently extended to layered periodic structures by virtue of the Bloch-Floquet theorem. Starting from a modal expansion of the fields in each layer, these techniques lead to a representation of the layer and of the transition between two adjacent layers in terms of an equivalent network; the different networks are then cascaded to represent the entire structure. The form of the matrix describing this network depends on the type of parameter used to characterize the structure performances: for example the generalized scattering matrix (GSM) [7], the generalized impedance matrix (GIM) and the generalized admittance matrix (GAM) [8].

In a GSM-based representation, the problem is typically solved separately for each incident Floquet harmonic, by calculating the corresponding induced current and scattering parameters [7]. A more efficient formulation proposed in [4], [9], [10] allows the computation of the scattering parameters for all incident modes at once.

In many cases, the calculation of the GIM or GAM equivalent representation is the goal of the analysis. An appealing characteristic of these representations is that they can be directly inter- 
preted in terms of physical parameters of the represented structure. For example, in [4] a GSM formulation was presented to analyse a microstrip phased array antenna and, to obtain the antenna input impedance, from the calculated GSM the GAM of the slot/microstrip-line transition was derived. In a recent paper [11], it has been shown that the equivalent admittance matrix of a dipole FSS is a useful tool to investigate the dispersion properties of the surface waves modes, and it can be directly linked to the phase behavior of the reflection coefficient. In [8], [12] a zero-thickness planar grating was characterized in terms of GAM and GIM, but only the two-dimensional cases were considered.

This paper describes an efficient method to calculate the GIM or GAM representation of multilayer planar structures, consisting of infinite periodic waveguide phased arrays and FSSs, composed by zero-thickness apertures and patches with an arbitrary shape, arranged in a rectangular or triangular lattice. The method, further referred here as the multimode equivalent network approach based on the integral equation formulation (IEMEN), was originally proposed for waveguide junctions [13], [14] and conformal waveguide arrays [15], and is extended here to the analysis of FSSs.

It was already pointed out in [16] that the GAM and GIM representations encounter a stability problem when matrices with a large number of entries are cascaded. To prevent this problem, additional operations should be undertaken to derive from the complete matrix a reduced form corresponding only to the interacting modes between adjacent discontinuities. In this respect, the IEMEN method allows directly calculating an equivalent impedance matrix representation in terms of these modes only. The formulation of the problem is centred around a single integral equation (IE) with a fixed reduced kernel and multiple forcing terms. In this paper, the IEMEN formulation is presented for both patch- and aperture-based FSSs. In [11] it was shown that poles and zeros of the FSS admittance matrix are slowly varying functions of the frequency and can be interpolated. This property becomes explicit in the IEMEN formulation, thanks to the adopted expression of the reduced spectral periodic Green's Function. The solution of a reduced kernel IE was already presented in [4] to derive a GSM representation of slot junctions, but the properties of this kernel were not further investigated.

The presented IEMEN method is suited to be very efficiently interpolated in frequency, so that results on large bandwidths can be rapidly calculated. Moreover, with respect to other techniques based on the derivation of microwave network representations, it offers several other advantages that will be discussed in detail in the paper.

The paper is structured as follows. In Section II, the theoretical formulation of the IEMEN method is briefly recalled. In Section III and Section IV the formulation is applied to the analysis of planar FSSs. In Section V considerations on the numerical implementation of the method with some emphasis on the solution of the pertinent IE are reported. Finally, validation of the method is performed in Section VI through comparison with results available in literature. Conclusions are drawn in Section VII.

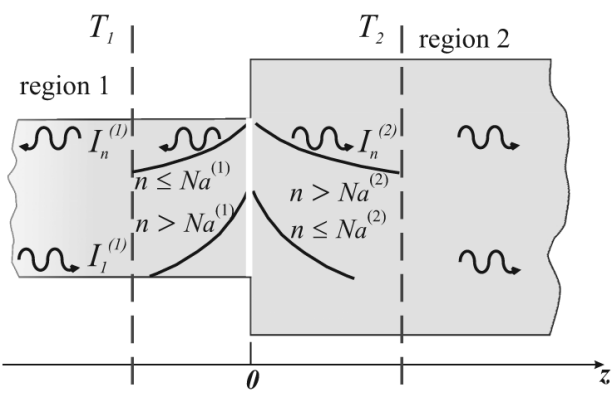

Fig. 2. Accessible and localized modes in a waveguide junction.

\section{MEN FORMULATION FOR THE TRANSITIONS}

To present the idea underlying the IEMEN approach, we consider the multilayer structure depicted in Fig. 1. The propagation of the electromagnetic field in the presence of an infinite periodic phased array can be described as the propagation in an equivalent waveguide with cross section equal to the array unit cell: the phase shift wall waveguide (PSWW) [17], satisfying the periodic boundary conditions dictated by the array. For each uniform waveguide region ( $z$-invariant regions between discontinuities) and each discontinuity a representation in terms of a multimode impedance or admittance matrix is derived.

The first step consists in expressing the fields in each region in terms of modes and identifying their pertinent transmission-line representations (along $z$ ). In the special case of Fig. 1, these discontinuities are localized at the planes $z=0, z=z_{1}, z=z_{2}$, $z=z_{3} . z$-invariant regions can be treated as uniform waveguide sections and the calculation of the corresponding equivalent network is a canonical problem [14].

In the second step, the concept of accessible and localized modes is exploited. Accessible are the modes that contribute to the interaction between adjacent discontinuities, not only the propagating ones, but also modes below cutoff. All remaining higher order modes are further indicated as localized [18]. As an illustration, Fig. 2 shows the junction between two uniform waveguide regions, indicated as regions 1 and 2 . In the case of an FSS, they are the PSWWs corresponding to the unit cell containing the patch or the aperture, located at $z=0$. The terminal planes $T_{1}$ and $T_{2}$ define the boundaries between the discontinuity region (namely, the region containing the junction) and the two waveguides. If the structure consists of more than two waveguides, the terminals are located in between the adjacent junctions. Otherwise they can be chosen arbitrarily far from the current junction at $z=0$. A complete representation of the transverse field at the junction requires taking into account an infinite number of evanescent modes. Instead, the field distribution at the two terminal planes can be described, within a certain accuracy, in terms of a finite number of modes (accessible modes). The accuracy of this description is specified by setting a maximum value of attenuation of the modes when they reach the terminal planes.

In particular, if $N_{a}^{(1)}$ and $N_{a}^{(2)}$ accessible modes are sufficient to obtain the desired accuracy at the terminal planes $T_{1}$ and $T_{2}$ in Fig. 2, the modal expression of the electric and magnetic fields [19] can be correspondingly separated into two contributions: 
one with the accessible modes (the first $N_{a}^{(\delta)}$ modes, with $\delta=1$, 2 indicating one of the two regions), and one with all remaining localized modes

$$
\begin{aligned}
\mathbf{E}_{t}^{(\delta)}(\mathbf{r})= & \sum_{i=1}^{N_{a}^{(\delta)}} V_{i}^{(\delta)}(z) \mathbf{e}_{i}^{(\delta)}\left(\mathbf{r}_{\boldsymbol{t}}\right) \\
& +\sum_{i=N_{a}^{(\delta)}+1}^{\infty} V_{i}^{(\delta)}(z) \mathbf{e}_{i}^{(\delta)}\left(\mathbf{r}_{\boldsymbol{t}}\right) \\
\mathbf{H}_{t}^{(\delta)}(\mathbf{r})= & \sum_{i=1}^{N_{a}^{(\delta)}} I_{i}^{(\delta)}(z) \mathbf{h}_{i}^{(\delta)}\left(\mathbf{r}_{\boldsymbol{t}}\right) \\
& +\sum_{i=N_{a}^{(\delta)}+1}^{\infty} I_{i}^{(\delta)}(z) \mathbf{h}_{i}^{(\delta)}\left(\mathbf{r}_{\boldsymbol{t}}\right) .
\end{aligned}
$$

These two contributions will be treated differently in solving the IE, as outlined in the next sections.

By virtue of the uniqueness property of the electromagnetic field, if the region enclosed by the terminal planes is source-free, the knowledge of either the tangential electric or the tangential magnetic field at the terminal planes characterizes uniquely the electromagnetic field within that region [20]. Therefore, once the accuracy in the calculation of the field at the terminal planes is set and the pertinent accessible modes have been identified, the corresponding modal coefficients $\left(V^{(\delta)}\right.$ and $\left.I^{(\delta)}\right)$ in (1) are the fundamental unknowns of the problem, in the sense that all the fields in the region delimited by the terminal planes can be expressed, with the specified accuracy, in terms of these modal coefficients only. These modes define the ports of the equivalent network. As we approach the transition at $z=0$, more modes are needed to obtain a complete field representation at that section (all the localized ones), but their contribution to the field representation at the terminal planes still depends on the fundamental unknowns. This is actually the basic idea of the IEMEN method. The method is explicitly described, for the FSS case, in the next section. Before presenting it, a few considerations are in order.

In case of an FSS-type of transition, the modal functions in (1) are Floquet waves and, since the tangential electric and magnetic fields are continuous at the interface between the two dielectric half spaces, if the unit cell is the same (same cross section for the two PSWWs in Fig. 2) the modal functions are the same in the two regions and the superscript $(\delta)$ may be dropped, (the explicit expressions of the Floquet vector modal functions $\mathbf{e}_{i}$ and $\mathbf{h}_{i}$ are given in the Appendix). The description of the fields of the terminal planes involves then $\max \left(N_{a}^{(1)}, N_{a}^{(2)}\right)$ modal voltages and the same number of modal currents, related by $\max \left(N_{a}^{(1)}, N_{a}^{(2)}\right)$ linear equations.

GAM/GIM-based methods, in their classical implementations, may encounter a stability problem. The problem has been well outlined in [16] for the case of a branch guide coupler studied as cascade of the GAM corresponding to waveguides and stubs. Normally, the GAM is calculated for a large number of entries, corresponding to the modes (accessible and localized) used in the representation of the fields at the junction,

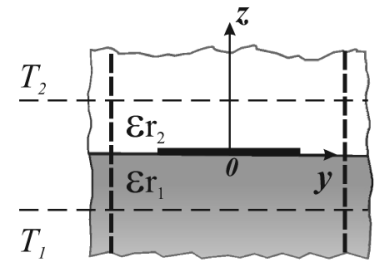

(a)

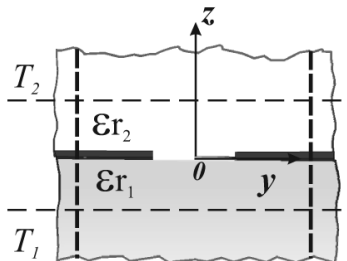

(b)
Fig. 3. Unit cell of a planar FSS, seen as (a) a patch transition for the patch formulation and (b) a slot transition for the aperture formulation.

according to (1). If in this representation are included also evanescent modes that arrive very attenuated to the next junction, the corresponding entries of the waveguide admittance matrix are close to zero leading to a not invertible matrix. As suggested in [16], the problem can be circumvented by calculating a reduced form of this matrix, which contains only the accessible modes. Moreover, it has the advantage of using less computer memory. Usually, to derive the reduced form of a GAM or GIM representation, the input and output ports of the equivalent network corresponding to the non-accessible modes are closed with the characteristic admittances/impedances. The numerical implementation of this operation implies performing an additional matrix inversion (with size equal to the number of localized modes) [16].

The IEMEN method avoids this extra operation and directly obtains a reduced GIM- or GAM-based network by solving a reduced kernel IE. Analogous considerations hold also for the GAM-based analysis of an FSS-type of transition (FSS cascaded to a PSWW).

\section{PATCh-BASED FSS}

As shown in Fig. 3, for a general array of zero thickness patches, two complementary IEs can be obtained, in which either the induced currents on the patches or the tangential electric fields in the complementary apertures are treated as the unknowns of the problem. The choice depends on the shape of the patch/complementary aperture and on the polarization of the field [8], [9]. In the patch formulation, the problem is formalized in terms of an electric field integral equation (EFIE) and the unknown quantities are the induced electric surface currents on the patch. Equivalently, they can be seen as currents flowing in a generalized load connected in parallel to the transmission lines associated to the Floquet modes, defined above and below the FSS discontinuity. The corresponding representation of the transition is a shunt multimode admittance network. In the aperture formulation instead, the problem is formalized in terms of a magnetic field integral equation (MFIE). In this case, the unknowns are the equivalent magnetic currents (tangential electric field in the aperture), and the transition is represented in terms of a shunt multimode impedance network. In the following, we will explicitly derive the equivalent network representations for these transitions. The patch formulation is described in detail in this section, while the aperture formulation is briefly presented in Section IV. 


\section{A. Reduced Integral Equation}

Fig. 3(a) shows a cross sectional view of the unit cell of an infinite doubly periodic distribution of metallic patches between two different dielectric half spaces. In each elementary cell of the array, the total tangential electric field must vanish on the surface of the patches, which are assumed perfectly conducting and infinitesimally thin. As described in Section II, the representation in terms of Floquet vector mode functions, $\mathbf{e}_{i}$ and $\mathbf{h}_{i}$, is the same in the two half spaces. Accordingly, the boundary condition for the tangential electric field on the patch located at the origin of the reference system, $(z=0)$, can be expressed as

$$
\mathbf{E}_{t}\left(\mathbf{r}_{t}\right)=\sum_{i=1}^{\infty} V_{i} \mathbf{e}_{i}\left(\mathbf{r}_{t}\right)=0, \quad \mathbf{r}_{t} \in S_{p}
$$

where $\mathbf{r}_{t}=x \hat{\mathbf{x}}+y \hat{\mathbf{y}}$ identifies a point on the patch, with respect to the Cartesian reference system indicated in Fig. 3(a), and $S_{p}$ is the surface of the patch. Note that with the index $(i)$ we have indicated both TE and TM modes. Equation (2) is valid in both regions. The modal voltages are here evaluated at the discontinuity and, for the sake of simplicity, we have indicated $V_{n}(z=0)=V_{n}$. The first step in the definition of the Multimode Equivalent Network of the transition is the identification of the subsets of Floquet modes $N_{a}^{(1)}$ and $N_{a}^{(2)}$ that are considered accessible in the two dielectric half spaces, with respect to the chosen pair of terminal planes. The corresponding modal voltages $V_{n}$ are the fundamental unknowns of the scattering problem. In the present case, the number of fundamental unknowns is equal to the maximum number of accessible modes between those relevant at the terminal plane $T_{1}$ and those at the terminal plane $T_{2}: N_{a}=\max \left(N_{a}^{(1)}, N_{a}^{(2)}\right)$. Consequently, it is appropriate to express the equivalent electric current distribution on the metallic conductors $\mathbf{J}(\mathbf{r})=\hat{\mathbf{z}} \times\left(\mathbf{H}_{t}^{(2)}(\mathbf{r})-\mathbf{H}_{t}^{(1)}(\mathbf{r})\right)$, which is the unknown physical quantity of the problem, in terms of the first $N_{a}$ modal voltages, with $N_{a}$ suitable functions $\mathbf{j}_{i}\left(\mathbf{r}_{t}\right)$ as unknown vectorial coefficients to be determined

$$
\mathbf{J}\left(\mathbf{r}_{t}\right)=\sum_{i=1}^{N_{a}} V_{i} \mathbf{j}_{i}\left(\mathbf{r}_{t}\right)
$$

We can also write the total transverse electric field in (2) as a superposition of accessible and localized contributions

$$
\begin{aligned}
\mathbf{E}_{t}\left(\mathbf{r}_{t}\right) & =\mathbf{E}_{t_{a}}\left(\mathbf{r}_{t}\right)+\mathbf{E}_{t_{n a}}\left(\mathbf{r}_{t}\right) \\
& =\sum_{i=1}^{N_{a}} V_{i} \mathbf{e}_{i}\left(\mathbf{r}_{t}\right)+\sum_{i=N_{a}+1}^{\infty} V_{i} \mathbf{e}_{i}\left(\mathbf{r}_{t}\right)
\end{aligned}
$$

where we are taking into account both TE and TM modes. Note that the field associated to the localized modes $\mathbf{E}_{t_{n a}}\left(\mathbf{r}_{t}\right)$ does not interact with any other part of the circuit (it does not contribute to the field at the terminal planes) and stores the reactive energy. In view of this, it is convenient to define the non-accessible $(n a)$ constituent of the infinite array dyadic Green's function (GF) for the electric field, $\overline{\mathbf{G}}_{n a}^{e}$, as derived in the Appendix, (34a)

$$
\overline{\mathbf{G}}_{n a}^{e,(\rho)}\left(\mathbf{r}_{t} ; \mathbf{r}_{t}^{\prime}\right)=-\sum_{i=N_{a}+1}^{\infty}\left(Z^{(\rho)}\left(\mathbf{k}_{t_{i}}\right) \mathbf{e}_{i}^{(\rho)}\left(\mathbf{r}_{t}\right) \mathbf{e}_{i}^{(\rho) *}\left(\mathbf{r}_{t}^{\prime}\right)\right)
$$

where $\overline{\mathbf{G}}_{n a}^{e,(\rho)}\left(\mathbf{r}_{t} ; \mathbf{r}_{t}^{\prime}\right)$ is valid for electric source and electric observation located at $z=z^{\prime}=0$ and $\rho=T E, T M . Z\left(\mathbf{k}_{t_{i}}\right)=$ $Z\left(\mathbf{k}_{t_{i}}, 0\right)$ are the modal impedances at the section $z=0$, obtained by connecting in parallel the input impedances on the left $Z_{1 i}\left(\mathbf{k}_{t_{i}}, 0\right)$ and on the right $Z_{2 i}\left(\mathbf{k}_{t_{i}}, 0\right)$ of the FSS

$$
Z_{i}\left(\mathbf{k}_{t_{i}}, 0\right)=\frac{Z_{1 i}\left(\mathbf{k}_{t_{i}}, 0\right) Z_{2 i}\left(\mathbf{k}_{t_{i}}, 0\right)}{Z_{1 i}\left(\mathbf{k}_{t_{i}}, 0\right)+Z_{2 i}\left(\mathbf{k}_{t_{i}}, 0\right)}
$$

By using this concept, $\mathbf{E}_{t_{n a}}\left(\mathbf{r}_{t}\right)$ can be expressed as

$$
\mathbf{E}_{t_{n a}}\left(\mathbf{r}_{t}\right)=\sum_{i=N_{a}+1}^{\infty} V_{i} \mathbf{e}_{i}\left(\mathbf{r}_{t}\right)=\int_{S p} \overline{\mathbf{G}}_{n a}^{e}\left(\mathbf{r}_{t} ; \mathbf{r}_{t}^{\prime}\right) \cdot \mathbf{J}\left(\mathbf{r}_{t}^{\prime}\right) d \mathbf{r}_{t}^{\prime} .
$$

To simplify the notation, we have omitted the explicit indication of the mode type. Note that, for the case of a stacked FSS, the IEMEN formulation is applied to each patch transition separately and $\mathbf{J}\left(\mathbf{r}_{t}^{\prime}\right)$ indicates the equivalent current distribution pertinent to the patch layer under analysis. Inserting (7) and (4) in (2), the following EFIE is obtained for both TE and TM modes:

$$
\sum_{i=1}^{N_{a}} V_{i} \mathbf{e}_{i}\left(\mathbf{r}_{t}\right)=-\int_{S p} \overline{\mathbf{G}}_{n a}^{e}\left(\mathbf{r}_{t} ; \mathbf{r}_{t}^{\prime}\right) \cdot \mathbf{J}\left(\mathbf{r}_{t}^{\prime}\right) d \mathbf{r}_{t}^{\prime}
$$

where $\mathbf{r}_{t} \in S_{p}$. This equation relates the accessible modes to the equivalent currents on the patch. Inserting the expression for the unknown equivalent currents (3) into (8) leads to

$$
\sum_{i=1}^{N_{a}} V_{i} \mathbf{e}_{i}\left(\mathbf{r}_{t}\right)=-\sum_{i=1}^{N_{a}} V_{i} \int_{S p} \overline{\mathbf{G}}_{n a}^{e}\left(\mathbf{r}_{t} ; \mathbf{r}_{t}^{\prime}\right) \cdot \mathbf{j}_{i}\left(\mathbf{r}_{t}^{\prime}\right) d \mathbf{r}_{t}^{\prime} .
$$

By virtue of the nature of the accessible modal voltages, this equation is valid for all possible values of $V_{i}$, with $i=1 \ldots N_{a}$. As a consequence, we can equate the coefficients of $V_{i}$ on both sides of the equation and obtain the IE

$$
\mathbf{e}_{i}\left(\mathbf{r}_{t}\right)=-\int_{S p} \overline{\mathbf{G}}_{n a}^{e}\left(\mathbf{r}_{t} ; \mathbf{r}_{t}^{\prime}\right) \cdot \mathbf{j}_{i}\left(\mathbf{r}_{t}^{\prime}\right) d \mathbf{r}_{t}^{\prime} \quad \mathbf{r}_{t} \in S_{p}
$$

where $i=1, \ldots, N_{a}$. The IEMEN formulation resorts to a single IE with, as forcing terms, the extracted Floquet modes and with a reduced kernel containing the non-accessible GF, $\overline{\mathbf{G}}_{n a}^{e}$, rather than the standard periodic array GF.

Since the extracted low-order Floquet modes are the most frequency dependent ones, $\overline{\mathbf{G}}_{n a}^{e}$ has a weaker frequency dependence than the standard periodic GF. Thus, as a first consequence of the present formulation, the larger the number of accessible modes, the slower is the variation of each solution $\mathbf{j}_{i}$ with the frequency.

\section{B. Multimode Equivalent Network}

The unknown functions $\mathbf{j}_{i}\left(\mathbf{r}_{t}^{\prime}\right)$, obtained by solving IE (10), weighted by proper coefficients as in (3), provide a representa- 
tion of the equivalent current $\mathbf{J}\left(\mathbf{r}_{t}\right)$ and consequently of the total tangential magnetic field difference at the interface containing the patch $\left.-\hat{\mathbf{z}} \times \mathbf{J}\left(\mathbf{r}_{t}\right)=\left(\mathbf{H}_{t}^{(2)}(\mathbf{r})-\mathbf{H}_{t}^{(1)} \mathbf{r}\right)\right)$.

The Floquet expansion of the magnetic fields: $\mathbf{H}_{t}^{(\gamma)}(\mathbf{r})=$ $\sum_{m=1}^{\infty} I_{m}^{(\gamma)} \mathbf{h}_{m}\left(\mathbf{r}_{t}\right)$, with $\gamma=1,2$, can then be invoked to derive a relation between the $N_{a}$ modal voltages and currents in input to the equivalent network that represents the transition

$$
\sum_{m=1}^{\infty}\left(I_{m}^{(2)}-I_{m}^{(1)}\right) \mathbf{h}_{m}\left(\mathbf{r}_{t}\right)=-\hat{\mathbf{z}} \times \sum_{j=1}^{N_{a}} V_{j} \mathbf{j}_{j}\left(\mathbf{r}_{t}\right) .
$$

The terminal conditions are obtained by performing the integral projection of both sides of (11) on the set of accessible modes $\mathbf{h}_{i}\left(\mathbf{r}_{t}\right)$, with $i=1, \ldots, N_{a}$

$$
\begin{aligned}
\int_{\partial S}\left(\sum_{m=1}^{N_{a}}\left(I_{m}^{(2)}-I_{m}^{(1)}\right) \mathbf{h}_{m}\left(\mathbf{r}_{t}\right)\right. \\
\left.+\sum_{m=N_{a}+1}^{\infty}\left(I_{m}^{(2)}-I_{m}^{(1)}\right) \mathbf{h}_{m}\left(\mathbf{r}_{t}\right)\right) \cdot \mathbf{h}_{i}^{*}\left(\mathbf{r}_{t}\right) d \mathbf{r}_{t} \\
\quad=\int_{\partial S}\left(-\hat{\mathbf{z}} \times \sum_{j=1}^{M} V_{j} \mathbf{j}_{j}\left(\mathbf{r}_{t}\right)\right) \cdot \mathbf{h}_{i}^{*}\left(\mathbf{r}_{t}\right) d \mathbf{r}_{t}
\end{aligned}
$$

where $\partial S$ is the periodic cell surface. Using the orthogonality of the Floquet modes on the unit cell [21], and some straightforward algebraic manipulations, (12) results in the following terminal conditions:

$$
I_{i}=\sum_{j=1}^{N_{a}} V_{j} Y_{i j}, \quad i=1, \ldots, N_{a}
$$

where $I_{i}=I_{i}^{(1)}-I_{i}^{(2)}$ and

$$
Y_{i j}=\int_{\partial S} \mathbf{j}_{j}\left(\mathbf{r}_{t}\right) \cdot \mathbf{e}_{i}^{*}\left(\mathbf{r}_{t}\right) d \mathbf{r}_{t}, \quad j=1, \ldots, N_{a} .
$$

Thus, the equivalent network for the patch transition, obtained by applying the IEMEN formulation, is a multimode admittance in parallel to the transmission lines associated to the accessible modes in the two different dielectric regions. This network is depicted in Fig. 4. It is worth to note that, if $N^{(1)}<N^{(2)}$, then $I_{i}^{(1)}=-Y_{0 i}^{(1)} V_{i}$ for $i>N^{(1)}$, which means that the corresponding $N^{(2)}-N^{(1)}$ ports of the equivalent network are closed with the characteristic admittance $Y_{0 i}^{(1)}$ of those modes. The same holds when $N^{(2)}<N^{(1)}$ at the other side of the network.

Since the solutions $\mathbf{j}_{i}$ are slowly varying functions of the frequency, the same holds for the terms $Y_{i j}$. While this is a general property of the GAM, which remains valid no matter how the matrix is obtained (it has also been observed in [11]), the present derivation makes it explicitly evident.

\section{APERTURE-BASED FSS}

The problem of an aperture-based FSS, obtained from a thick metal plate, can be formulated as a discontinuity problem between a waveguide array and free space. It has already been

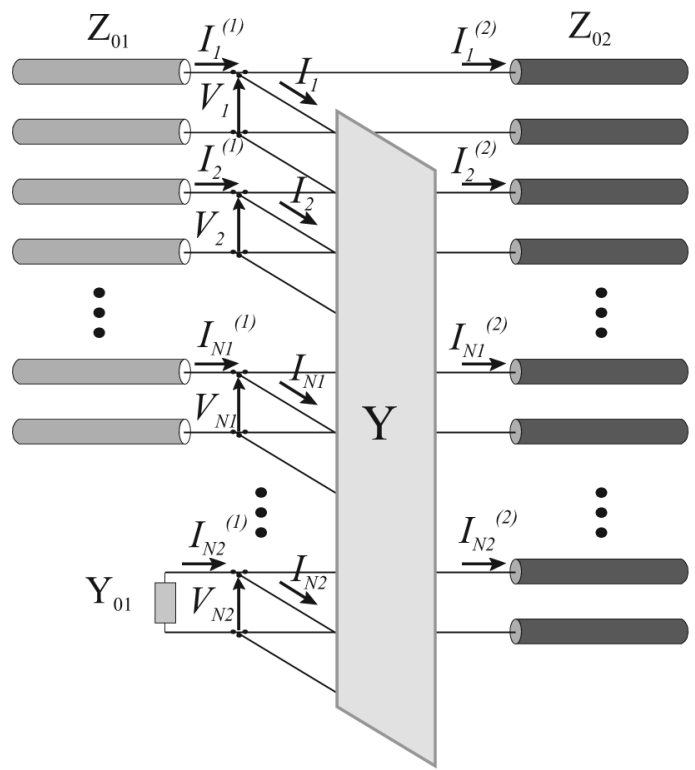

Fig. 4. Multimode equivalent network of a capacitive FSS, made of metal dipoles, for which the IEMEN method with the patch formulation is used; the matrix elements are defined in (14).

treated with the IEMEN method for a cylindrical geometry [15] and extended to the planar geometry in [22]. The application of the procedure described in Section III for the patch-based FSS to the case of infinitesimally thin screen is straightforward and leads to the following reduced kernel IE:

$$
\mathbf{h}_{i}\left(\mathbf{r}_{t}\right)=-\int_{S_{a}} \overline{\mathbf{G}}_{n a}^{h}\left(\mathbf{r}_{t} ; \mathbf{r}_{t}^{\prime}\right) \cdot \mathbf{m}_{i}\left(\mathbf{r}_{t}^{\prime}\right) d \mathbf{r}_{t}^{\prime}
$$

where $\mathbf{r}_{t} \in S_{a}$ (surface of the aperture), $i=1, \ldots, N_{a}$ and $\mathbf{m}_{i}\left(\mathbf{r}_{t}\right)$ are unknown vectorial functions. Note, that here

$$
\overline{\mathbf{G}}_{n a}^{h}\left(\mathbf{r}_{t} ; \mathbf{r}_{t}^{\prime}\right)=\left(\overline{\mathbf{G}}_{n a}^{h(1)}\left(\mathbf{r}_{t} ; \mathbf{r}_{t}^{\prime}\right)+\overline{\mathbf{G}}_{n a}^{h(2)}\left(\mathbf{r}_{t} ; \mathbf{r}_{t}^{\prime}\right)\right)
$$

is the sum of the non-accessible constituents of the infinite array periodic GF, for the case of magnetic source and magnetic observation point, given by (35) in the Appendix. The corresponding equivalent network is a multimode impedance matrix analogous to the admittance matrix shown in Fig. 4 for the patch formulation, connected in parallel to the transmission lines associated to the accessible modes. The interested reader can refer to [22] for a detailed derivation.

\section{SOLUTION OF THE INTEGRAL EQUATIONS}

The peculiar aspect of IEs (10) and (15) is the reduced kernel, i.e., the extraction of the accessible modes from the complete GF. The IE can be solved numerically for the currents, with the aid of the Method of Moment (MoM) [23]. In particular, in IE (10) the unknowns $\mathbf{j}_{i}\left(\mathbf{r}_{t}\right)$ have been expanded in terms of $N_{f}$ sub-domain functions $\mathbf{f}_{k}\left(\mathbf{r}_{t}-\mathbf{r}_{t_{k}}\right)$, piecewise linear (PWL) or piecewise sinusoidal (PWS), located around $\mathbf{r}_{t_{k}} \equiv\left(x_{k}, y_{k}\right)$ for $k=1, \ldots, N_{f}$

$$
\mathbf{j}_{i}\left(\mathbf{r}_{t}\right)=\sum_{k=1}^{N_{f}} \beta_{k}^{(i)} \mathbf{f}_{k}\left(\mathbf{r}_{t}-\mathbf{r}_{t_{k}}\right)
$$


where $\beta_{k}^{(i)}$ are weights to be determined by solving the IE. Applying the MoM with Galerkin's procedure to (10) and substituting this expression of the unknowns leads to

$$
\begin{array}{r}
\int_{S_{p}} \mathbf{e}_{i}\left(\mathbf{r}_{t}\right) \cdot \mathbf{f}_{l}\left(\mathbf{r}_{t}-\mathbf{r}_{t_{l}}\right) d \mathbf{r}_{t}=-\sum_{k=1}^{N_{f}} \beta_{k}^{(i)} \iint_{S p} \mathbf{f}_{k}\left(\mathbf{r}_{t}^{\prime}-\mathbf{r}_{t_{k}}\right) \\
\cdot \overline{\mathbf{G}}_{n a}^{e}\left(\mathbf{r}_{t} ; \mathbf{r}_{t}^{\prime}\right) \cdot \mathbf{f}_{l}\left(\mathbf{r}_{t}-\mathbf{r}_{t_{l}}\right) d \mathbf{r}_{t}^{\prime} d \mathbf{r}_{t}
\end{array}
$$

where $i=1, \ldots, N_{a}$ and $l=1, \ldots, N_{f}$. Using (28) from the Appendix, the IE (18) becomes

$$
\begin{array}{r}
\mathbf{e}_{i}^{\prime} \cdot \tilde{\mathbf{f}}_{l}\left(-\mathbf{k}_{t_{i}}\right) e^{-j\left(\mathbf{k}_{t_{i}} \cdot \mathbf{r}_{t_{l}}\right)}=\sum_{k=1}^{N_{f}} \beta_{k}^{(i)}\left[-\frac{1}{d_{x} d_{y}} \sum_{p=N_{a}+1}^{\infty} \tilde{\mathbf{f}}_{l}\left(-\mathbf{k}_{t p}\right)\right. \\
\left.\cdot \tilde{\overline{\mathbf{G}}}\left(\mathbf{k}_{t p}\right) \cdot \tilde{\mathbf{f}}_{k}\left(\mathbf{k}_{t p}\right) e^{-j \mathbf{k}_{t p} \cdot\left(\mathbf{r}_{t_{l}}-\mathbf{r}_{t_{k}}\right)}\right]
\end{array}
$$

where the quantities with “ " are Fourier transforms of the corresponding quantities without $\sim$ and where $\tilde{\overline{\mathbf{G}}}^{e}\left(\mathbf{k}_{t p}\right)=\tilde{\overline{\mathbf{G}}}^{e}\left(\mathbf{k}_{t p}, 0\right)$ for the sake of simplicity. IE (19) is solved for $\beta_{k}^{(i)}$ for each forcing term $(i)$, and then the corresponding equivalent currents in (17) are substituted in (14) to determine each raw of the admittance matrix Y. Once the equivalent GAM or GIM of each transition and length of uniform waveguide is calculated, they are cascaded using the technique described in [24] to derive the matrix representation of the whole multilayer structure. From this, the derivation of the corresponding scattering parameters is straightforward.

Compared to a standard IE, where all Floquet modes contribute to the kernel, in case of reduced kernel it is observed that:

1) the MoM matrix elements might need to be calculated with higher accuracy;

2) the MoM matrix elements can be interpolated in frequency over larger intervals.

These two aspects will be treated in more detail in the following sections.

\section{A. Convergence of the MoM Solution}

In the design of a device that should exhibit a steep frequency filtering function over a wide range of angular incidences, it is usually needed to consider the cascade of several periodic surfaces. Moreover, to reduce the angle dependence, they are sandwiched between slabs of dielectric materials with high permittivity [1]. If the IEMEN approach is applied to characterize this multilayer structure, a large number of accessible modes has to be taken into account.

The corresponding IE has spatially fast varying forcing fields (the accessible modes) and requires a large number of unknowns to correctly represent the behavior of the currents induced on the patches. As a consequence, a large number of $p$ terms has to be summed in (19) to achieve convergence, with respect to a given relative precision.

The problem of relative convergence is common to all IE-based methods used to study the scattering of an FSS under plane-wave incidence and it is well explained in [25]. However, in the present formulation the extraction of the accessible

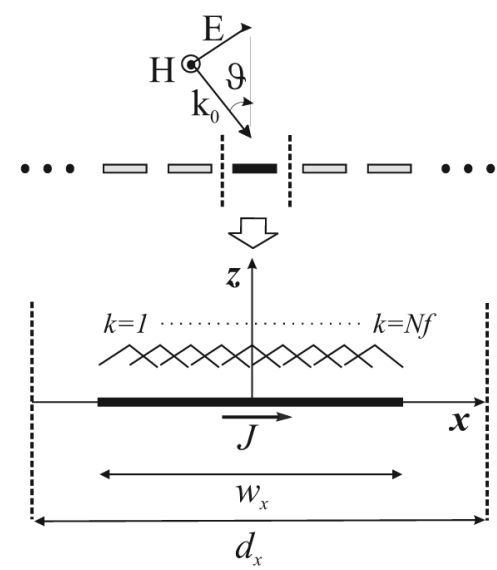

Fig. 5. Geometry of the investigated two-dimensional problem: infinite periodic array of conducting strips under TM plane-wave incidence.

modes from the kernel plays an important role in evaluating the convergence of the MoM analysis. In fact, the forcing terms in (10) are the electric fields associated to those same modes which have been extracted from the kernel. Accordingly, it is difficult for the unknown electric current on the patch to match the forcing fields. In particular, it results that the larger is the number of modes extracted from the kernel, the higher the condition number of the MoM matrix becomes.

To quantify this effect, we consider a simple two-dimensional geometry consisting of an infinite periodic array of metallic strips in free space. The period is $d_{x}=0.6 \lambda$ and the strips are infinitely long in the $y$-direction and have width $w_{x}=0.2 \lambda$. The incident wave is assumed to be TM polarized with respect to the $z$-axis, so that the magnetic field is entirely along the $y$-axis and the electric field has a component along the $x$-axis. Fig. 5 shows the investigated geometry.

The unknown functions $\mathbf{j}_{i}\left(\mathbf{r}_{t}\right)$ in (10) have been expanded in terms of $101 \mathrm{PWS}$ functions, with length $w_{x} / 102 * 2=0.004 \lambda$. The purpose of this choice is to simulate the complexity of a realistic three-dimensional problem, concerning for example an FSS that contains crossing points (e.g., a crossed dipole or a loop), where a large number of basis functions is needed to properly characterize the continuity of the currents in these points.

The condition numbers of the MoM matrices that are obtained by solving this scattering problem with conventional complete kernel and with reduced kernel are shown in Fig. 6. Note that the condition number is calculated as the product of the norm of the matrix and the estimated norm of the inverse matrix. The MoM matrix elements for this example were calculated with relative precision of $e_{A}=10^{-6}$.

In abscissa, the case of complete kernel is plotted in correspondence of $N_{a}=0$, while, for a reduced kernel, $N_{a}=2 N+1$ indicates the number of modes extracted from the kernel, with $N=0,1,2, \ldots$. The graph shows for the complete kernel case and for the case of only one accessible mode $\left(N_{a}=1\right)$ a similar condition number: $n_{c}=100$.

The relative precision of the IE solution, $e_{J}$, can be approximately related to the relative precision of the calculated MoM matrix elements $e_{A}$ through the matrix condition number $n_{c}$ : $e_{J} \leq n_{c} e_{A}$ [26]. Since, as shown in Fig. 6, in case of reduced 


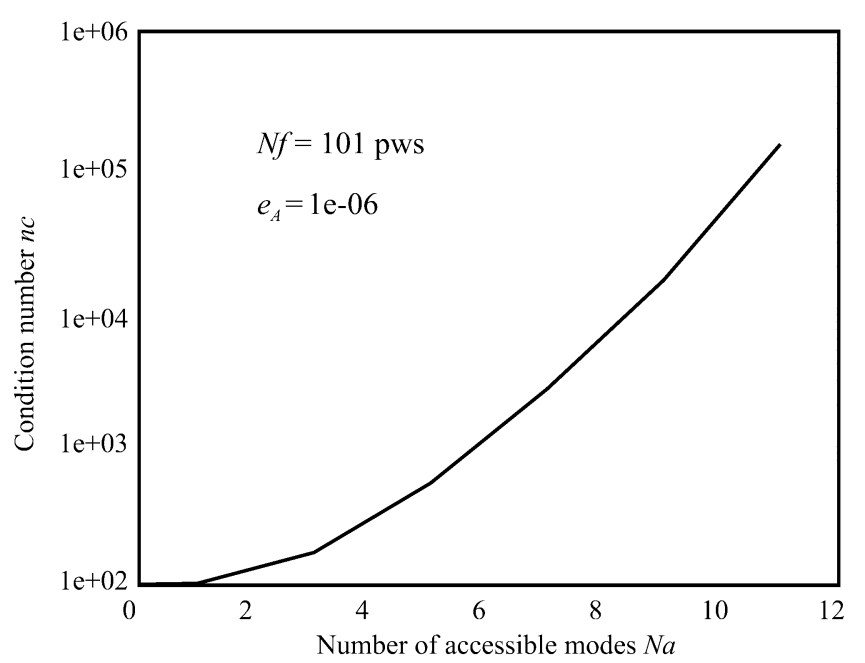

Fig. 6. Condition number of the MoM matrix obtained for different number of modes extracted from the kernel. In all cases, $101 \mathrm{pws}$ functions were used to expand the unknown functions $\mathbf{j}_{i}\left(\mathbf{r}_{t}\right)$. Note that $N_{a}=0$ corresponds to the complete kernel.

kernel the condition number is higher than that with a complete kernel, to guarantee a similar accuracy for the IE solution the elements of the MoM matrix might have to be calculated with an higher relative precision (lower $e_{A}$ ). This requires, for a certain expansion of the unknowns, to sum a larger number of contributions on the right-hand side of (19) (summation in $p$ ).

From Fig. 6, it is apparent that the worsening of the MoM matrix conditioning is a consequence of the adopted reducedkernel formulation. The same geometrical configuration does not give rise to conditioning problems when the equivalent electric currents on the dipoles are calculated by solving the standard IE (complete kernel), even if the incident electric fields are highly spatially varying functions. However, according to our experience, this convergence problem seldom occurs. Considering again the results for the simple strip case in Fig. 6, for a configuration including five accessible modes in the analysis (up to the indices \pm 2 ), the condition number grows to $n_{c}=500$. Note that this case corresponds, for a three dimensional problem to a maximum of 25 modes (supposing all possible combinations of indices are taken into consideration), which would cover the most typical configurations, where FSSs are sandwiched between dielectric layers designed to function as quarter-wavelength transformers [22].

Moreover, if one wishes to obtain the admittance matrix as a by product of the evaluation of the scattering matrix $\mathbf{S}$, calculated by means of a standard GSM method, a similar problem could be encountered. In the case of a single accessible mode, the relation between reflection coefficient and input admittance can be derived from its equivalent transmission line circuit

$$
Y_{i n}=Y_{0} \frac{1-\Gamma}{1+\Gamma}
$$

If $\Gamma=-1$ the input admittance $Y_{\text {in }}$ explodes. This observation can be extended to the multimodal case. The admittance matrix, normalized to the modal characteristic impedance, can be expressed as a function of the scattering parameters [27]

$$
\mathbf{Y}=(\mathbf{I}-\mathbf{S})(\mathbf{I}+\mathbf{S})^{-1}
$$

where $\mathbf{I}$ is the identity matrix.

When the dipole width $w_{x}$ is such that the i-th Floquet mode (even if of high order) is approaching resonance, the corresponding $S_{i i}$ parameter becomes close to -1 . The overall result is that the determinant of $(\mathbf{I}+\mathbf{S})$ assumes low values, which is symptomatic of an ill-conditioned matrix. To perform the matrix inversion in (21), the mutual coupling between basis and test functions should be calculated with an accuracy higher than that needed to evaluate the diagonal elements (associated to the self coupling). Thus, it seems that the conditioning problem is intrinsical to the evaluation of the generalized admittance (or impedance) matrix and not specifically related to the IEMEN method.

\section{B. Efficiency of the $3 D$ Implementation}

For the IEMEN method to be numerically handled, the evaluation of the series in the IE kernel has been accelerated by extracting the quasi-static contribution of the mutual coupling itself. The followed procedure, described in detail in [28], closely resembles the one presented by Wilton in [29] and is based on the Kummer transformation. However, while in [29] the acceleration method was developed for the case of scalar free-space GF, in [28] it has been extended to the dyadic multilayer GF. Moreover, geometrical symmetries are exploited in the calculation of the coupling between basis and test functions.

Despite the fact that the broad band analysis can benefit from the lower frequency variation of the MoM matrix elements, the solution of IE (10) may become cumbersome for a large number of accessible modes (see Fig. 6). This happens for example when the FSSs are printed on very thin dielectric slabs. To tackle this problem, instead of extracting from the kernel of the IE all the modes that reach the next dielectric stratification, we select only the propagating ones. For the remaining modes, we include the information about the finiteness of the dielectric directly in a multilayer spectral GF. This approach was already suggested in [7], [9], [10] for the GSM formulation and is implemented here in the reduced kernel IE obtained by applying the IEMEN method. Let us consider, for example, a patch FSS printed on a very thin board; a unit cell is shown in Fig. 7. We can write the left-hand side of (9) as dependent only on the first $N_{r}<N_{a}$ accessible modes (accessible at the terminal planes placed in region 2 and region 3 ) and include the remaining accessible modes in the kernel:

$$
\sum_{i=1}^{N r} V_{i} \mathbf{e}_{i}\left(\mathbf{r}_{t}\right)=-\sum_{i=1}^{N r}\left\{V_{i} \int_{S p} \overline{\mathbf{G}}_{n a(r)}^{e}\left(\mathbf{r}_{t} ; \mathbf{r}_{t}^{\prime}\right) \cdot \mathbf{J}\left(\mathbf{r}_{t}^{\prime}\right) d \mathbf{r}_{t}^{\prime},\right\}
$$

where

$$
\overline{\mathbf{G}}_{n a(r)}^{e}\left(\mathbf{r} ; \mathbf{r}^{\prime}\right)=-\sum_{i=N r+1}^{\infty}\left(Z_{i}\left(\mathbf{k}_{t_{i}}, 0\right) \mathbf{e}_{i}\left(\mathbf{r}_{t}\right) \mathbf{e}_{i}^{*}\left(\mathbf{r}_{t}^{\prime}\right)\right) .
$$




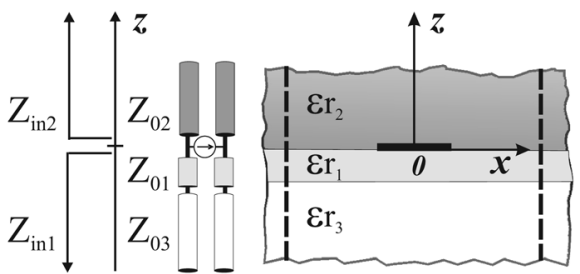

Fig. 7. Unit cell of a patch FSS etched on a thin dielectric slab and corresponding equivalent transmission line circuit.

The impedances $Z_{i}\left(\mathbf{k}_{t_{i}}, 0\right)$ are obtained by connecting in parallel the input impedances on the left and right of the FSS, $Z_{i n 1}$ and $Z_{i n 2}$, shown in the equivalent circuit at the side of Fig. 7. The GF now depends on the input impedance at the two sides of the transition. The same procedure can be followed to model the case of the patch etched on a semi-infinite dielectric half-space and covered by a thin dielectric slab.

\section{Frequency Dependence}

In spite of the worse conditioning with respect to a conventional kernel, there is an important aspect that makes convenient the numerical burden of the solution of the FSS problem via the present IEMEN method. The lower order modes are characterized by $k_{z}$ numbers that are more frequency dependent. In a $2 \mathrm{D}$ problem with normal incidence for instance, $k_{z n}=$ $\sqrt{k_{0}^{2}-\left(2 \pi n / d_{x}\right)^{2}}$. Once the lower order modes have been extracted, the MoM matrix elements present a weaker frequency dependence. As a consequence, the matrix entries can be interpolated over larger frequency domains, allowing a reduction of the overall calculation time for a broad band analysis. Fig. 8 shows the elements $i=1, j=1$ and $i=1, j=80$, of the MoM matrix calculated by means of the IEMEN method, in the frequency range $0.2-2 \mathrm{GHz}$. They are normalized to their maximum value, indicated between brackets in the figure, and multiplied by $k_{0}=2 \pi f \sqrt{\varepsilon \mu}$ to de-embed the frequency dependence. The same elements, obtained using the complete kernel, are also plotted for comparison. Note that the curve obtained for the complete kernel overlaps that corresponding to one accessible mode in Fig. 8(a) and those corresponding to one and three accessible modes in Fig. 8(b).

Grating lobes can be spotted at multiple frequencies of 500 $\mathrm{MHz}$, both for the complete kernel case and for the reduced kernel, if not all the modes that start propagating in the considered frequency range have been extracted. The different behavior with respect to the frequency is more evident for the off-diagonal elements of the MoM matrix, as shown in Fig. 8(b). In particular, it is observed that the larger the number of accessible modes, the easier the interpolation of the MoM matrix elements becomes. This property is particularly relevant for synthesis problems.

The solutions for the currents in the present IEMEN formulations provide, via simple projection onto the Floquet modes, the elements of the multimode admittance matrix. Since the currents present a slow variation with frequency, also the multimode admittance matrix elements are slowly varying with frequency. This was already observed by [11] but never explicitly demonstrated.

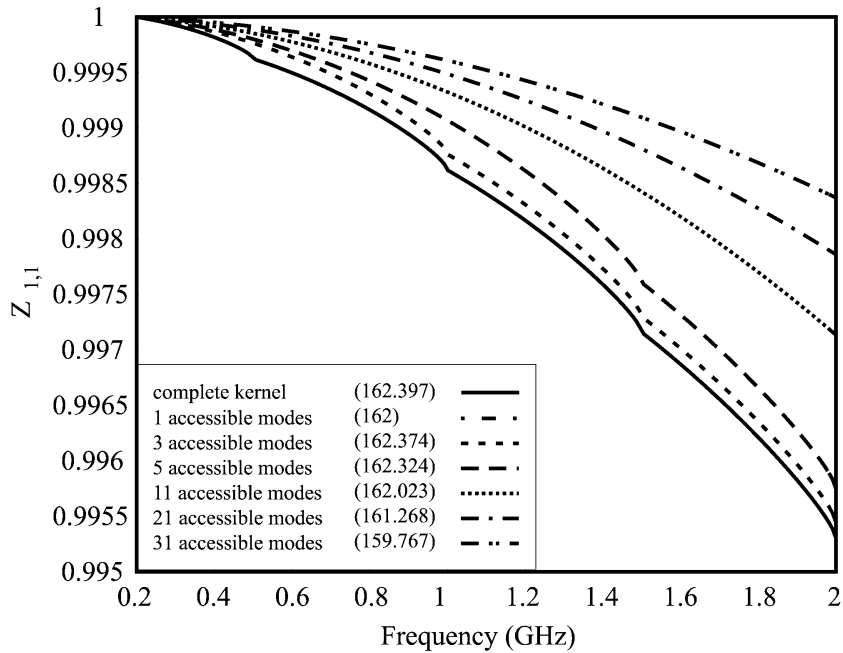

(a)

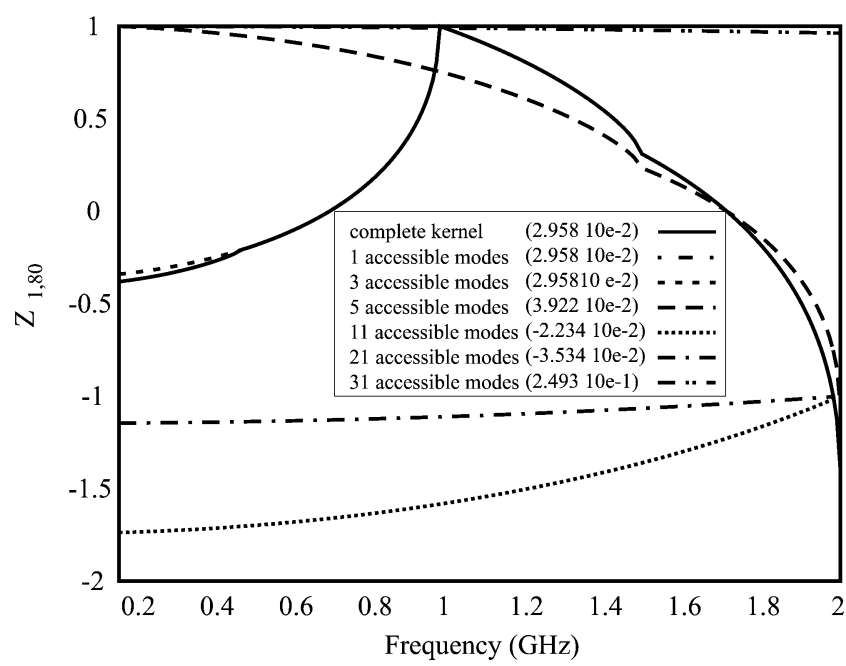

(b)

Fig. 8. Behavior of some elements of the MoM matrix as a function of the frequency: (a) $Z_{1,1}$ and (b) $Z_{1,80}$. The elements value is normalized to the maximum value assumed in the considered frequency range (indicated between brackets), and it is multiplied by $k_{0}=2 \pi f \sqrt{\varepsilon \mu}$.

\section{NUMERICAL RESULTS}

In this Section, we present some examples of the analysis capabilities of the MEN approach.

Fig. 9 shows the power reflection coefficient of an infinite array of crossed dipoles printed on a dielectric slab, versus the slab thickness indicated with $a$ (the unit cell and element geometry is shown in the inset). The simulations were performed for three different permittivities of the slab, when a TE polarized plane wave is impinging on the FSS plate almost at broadside $\left(\vartheta=1^{\circ}\right)$. The unknown currents were expanded in terms of 20 PWLs, and one accessible mode was included in the analysis. The MoM matrix elements were calculated with a relative precision of $e_{A}=10^{-6}$. The results based on the IEMEN method are displayed in Fig. 9, together with those presented in [7], obtained using a GSM approach. The agreement is remarkable.

Furthermore, we have analysed an FSS, proposed in [1], consisting of an infinite array of Jerusalem cross patches, arranged in square lattice, with cell dimension $5.091 \mathrm{~mm}$, embedded in a 


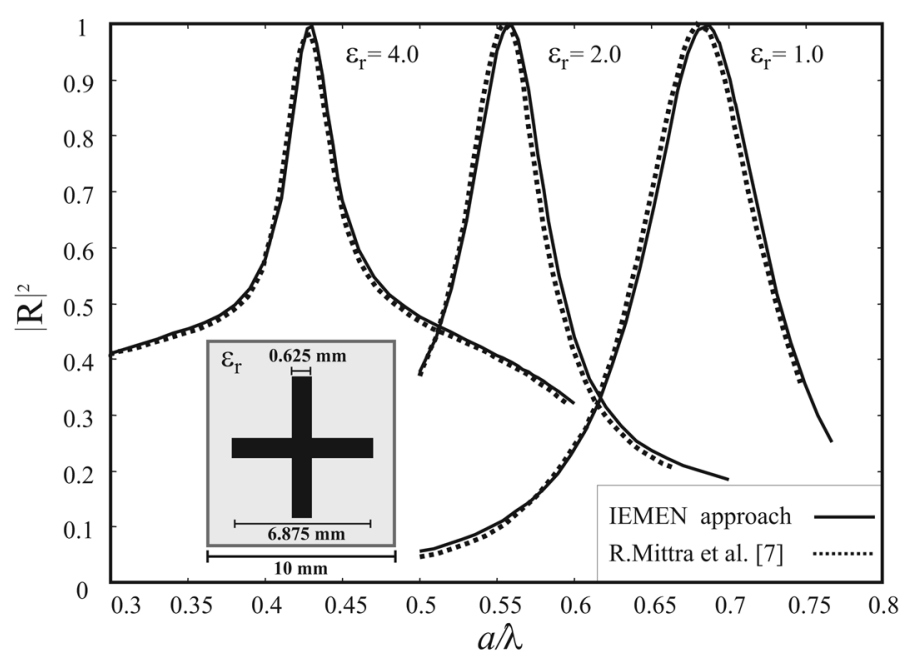

Fig. 9. Power reflection coefficient of a crossed-dipole FSS printed on a dielectric slab: comparison between the IEMEN simulation results and those reported by Mittra in [7].

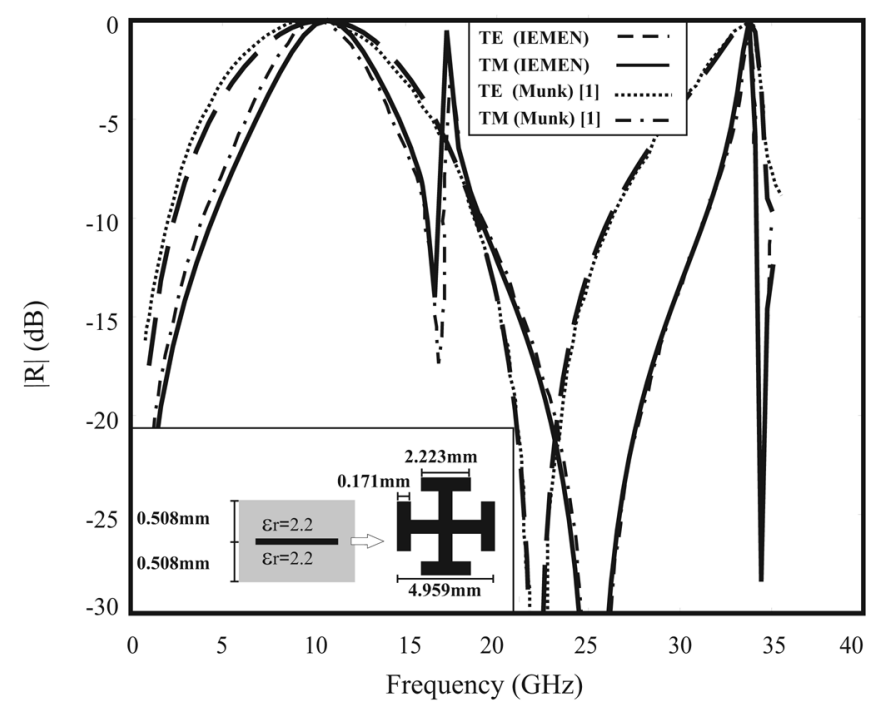

Fig. 10. Magnitude of the reflection coefficient of a Jerusalem cross FSS embedded in a dielectric slab: comparison between the IEMEN simulation results and those reported by Munk in [1].

dielectric slab of permittivity $\varepsilon_{r}=2.2$ and total thickness 1.016 $\mathrm{mm}$. The cross width is $0.171 \mathrm{~mm}$, the length is $4.959 \mathrm{~mm}$, while the transverse monopoles are $2.223 \mathrm{~mm}$ long, as shown in the inset of Fig. 10. The same figure shows the magnitude of the reflection coefficient for TE and TM plane wave incidence at an angle of $\vartheta=45^{\circ}$ on the E-plane, obtained by using 104 PWLs to expand the unknown currents and by extracting 14 accessible modes from the IE kernel. Excellent agreement is achieved with the results presented by Munk in [1]. Also in this case, the MoM matrix elements were calculated with a relative precision of $e_{A}=10^{-6}$.

Note that the structure could have been analysed by applying the procedure in Section V-B and considering only one accessible mode. A more significant case to show the effect of extracting accessible modes from the IE kernel is depicted in the inset of Fig. 11. It consists of the cascade of three equal screens

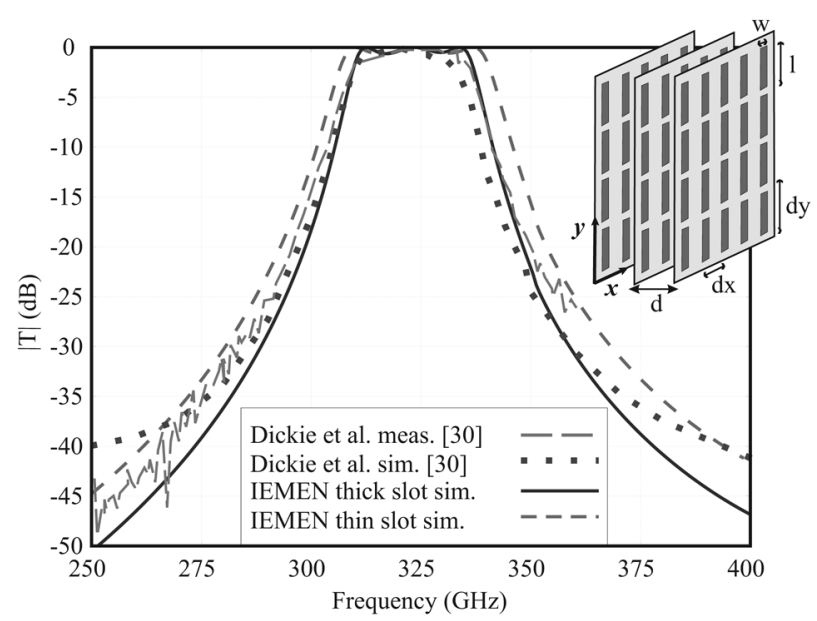

Fig. 11. Magnitude of the transmission coefficient of the 3-layer rectangular slot FSS described in [30]. Note that the curves have been normalized to the maximum value.

of rectangular slots arranged in a rectangular lattice and it is based on the structure described in [30]. The lattice dimensions are: $d x=0.49 \mathrm{~mm}$ and $d y=0.5 \mathrm{~mm}$. The slots are $0.46 \mathrm{~mm}$ long, $0.15 \mathrm{~mm}$ wide and they are cut on a metal plate $0.01 \mathrm{~mm}$ thick. The FSS is studied for TE plane wave incidence, with $\vartheta=45^{\circ}$ and $\varphi=90^{\circ}$. The structure has been simulated by treating the thick slots as lengths of rectangular waveguides; the first 6 accessible modes were extracted from the kernel and the same modes were also used as basis and test functions. Fig. 11 shows the simulated transmission coefficient together with the calculated result reported in [30] and the measured values. Note that while losses in the dielectric could be easily taken into account in the IEMEN-based tool, the ohmic losses have not been included yet, while they were introduced in the calculation results presented in [30]. These results have been normalized in Fig. 11 with respect to the maximum value. The transmission coefficient of this 3-layer FSS has been simulated also for the case of slots etched on an infinitesimally thin metal sheet, by solving (15). The equivalent magnetic currents on the slots have been meshed using 10 PWL functions and 3 accessible modes have been taken into account in the analysis $\left(\mathrm{TE}_{00}, \mathrm{TE}_{01}, \mathrm{TE}_{-10}\right)$. The result of this computation is also shown in Fig. 11. Note that extracting also the corresponding TM modes with the same indexes would not have changed the result, because the slots are very narrow and the magnetic current is mainly oriented along the $y$ direction.

Fig. 12 and Fig. 13 show the scattering and admittance matrix elements calculated for each FSS layer. The admittance elements have a smoother behavior and can be easily interpolated in the whole frequency range.

\section{CONCLUSION}

The IEMEN method, originally introduced to analyse multilayer waveguide structures, has been extended in this paper to FSSs consisting of infinitesimally thin metal elements. The method directly leads to a representation of the FSS in terms of a GIM or a GAM that depends only on the accessible modes, without need of additional matrix operations. 


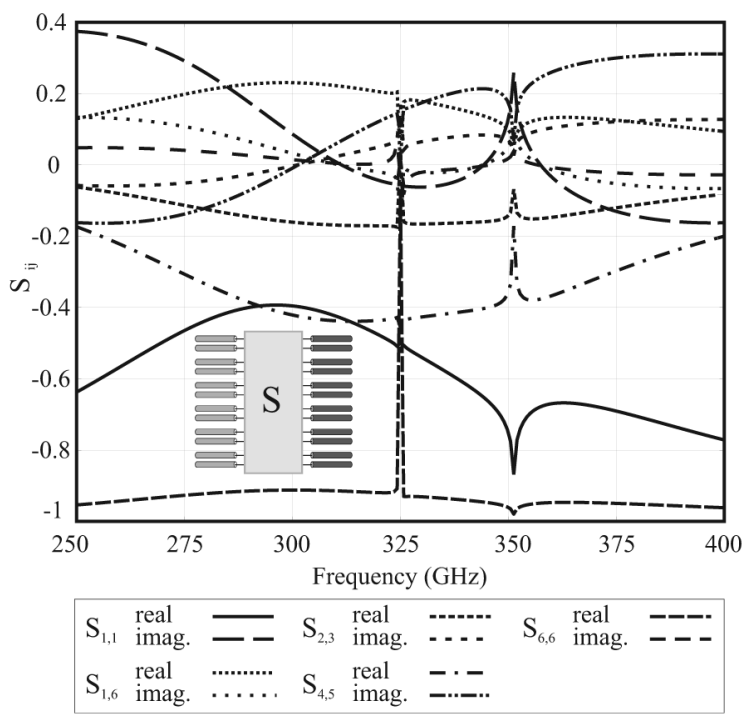

Fig. 12. Some elements of the equivalent scattering matrix of the FSS in Fig. 11, calculated by means of the IEMEN method with 3 accessible modes, for TE plane wave incidence.

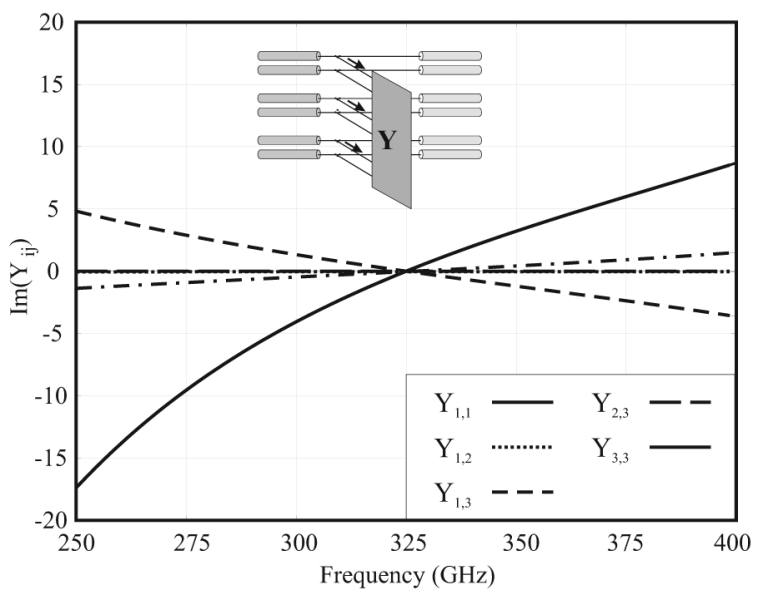

Fig. 13. Elements of the equivalent admittance matrix of the FSS in Fig. 11, calculated by means of the IEMEN method with three accessible modes, for TE plane wave incidence.

With respect to other methods based on equivalent network representation of a transition (waveguide or FSS), the IEMEN method offers two significant advantages.

- If a large number of accessible modes need to be considered, the evaluation of the equivalent admittance/impedance parameters might require high accuracy in the MoM matrix calculation to prevent ill-conditioning. While this problem appears in the IEMEN method directly in the solution of the IE, in the GSM approach it shows only at the end of the procedure, when deriving the GAM or GIM from the scattering matrix;

- Since MoM matrix and GAM entries are slowly varying function of the frequency, due to the extraction of the accessible modes from the IE kernel, the IEMEN method is particularly suitable for frequency interpolation.

The application of the IEMEN technique to the analysis of FSSs has been successfully validated versus results available in the open literature.
In many cases the calculation of the equivalent admittance or impedance matrices is the goal of the analysis and not just an intermediate step toward the scattering parameters. The formulation for the direct derivation of the (reduced) GAM and GIM is a useful theoretical tool, since these matrices are proportional to electromagnetic field quantities. For instance, in the evaluation of the Green's function of non periodic sources in the presence of periodic structures [31], a spectral representation of the fields can be directly obtained on the basis of multimode admittances or impedance matrices.

\section{APPENDIX \\ GREeN's FunCtion In TERMS OF FloQuet Modes}

Fig. 14 shows an infinite periodic array of patches, arranged in a rectangular lattice. The transverse components of the electric and magnetic fields in proximity of this structure can be expressed as a linear combination of Floquet vector mode functions, defined with respect to the $z$-axis. The vector mode functions for the TM modes are

$$
\begin{aligned}
\mathbf{e}_{i} & =\frac{1}{k_{t_{i}}} \frac{1}{\sqrt{d_{x} d_{y}}}\left(k_{x_{i}} \hat{\mathbf{x}}+k_{y_{i}} \hat{\mathbf{y}}\right) e^{-j\left(k_{x_{i}} x+k_{y_{i}} y\right)} \\
\mathbf{h}_{i} & =\frac{1}{k_{t_{i}}} \frac{1}{\sqrt{d_{x} d_{y}}}\left(-k_{y_{i}} \hat{\mathbf{x}}+k_{x_{i}} \hat{\mathbf{y}}\right) e^{-j\left(k_{x_{i}} x+k_{y_{i}} y\right)}
\end{aligned}
$$

and those for the TE modes are

$$
\begin{aligned}
\mathbf{e}_{i} & =\frac{1}{k_{t_{i}}} \frac{1}{\sqrt{d_{x} d_{y}}}\left(k_{y_{i}} \hat{\mathbf{x}}-k_{x_{i}} \hat{\mathbf{y}}\right) e^{-j\left(k_{x_{i}} x+k_{y_{i}} y\right)} \\
\mathbf{h}_{i} & =\frac{1}{k_{t_{i}}} \frac{1}{\sqrt{d_{x} d_{y}}}\left(k_{x_{i}} \hat{\mathbf{x}}+k_{y_{i}} \hat{\mathbf{y}}\right) e^{-j\left(k_{x_{i}} x+k_{y_{i}} y\right)}
\end{aligned}
$$

with

$$
\begin{aligned}
& k_{x_{i}}=k_{x_{0}}-\frac{2 \pi m}{d_{x}}=k_{0} \sin \vartheta \cos \varphi-\frac{2 \pi m}{d_{x}} \\
& k_{y_{i}}=k_{y_{0}}-\frac{2 \pi n}{d_{y}}=k_{0} \sin \vartheta \sin \varphi-\frac{2 \pi n}{d_{y}}
\end{aligned}
$$

and

$$
k_{t_{i}}=\sqrt{k_{x_{i}}^{2}+k_{y_{i}}^{2}} .
$$

In these expressions, the index $i$ refers to the $(m, n)$ th Floquet mode. The propagation of the $i$ th Floquet mode along the $z$-direction can be characterized in terms of an equivalent transmission line circuit. For the case depicted in Fig. 14, it consists of a current generator $I_{g}$ in parallel with the transmission lines representing the propagation of the Floquet mode in the two half-spaces, as shown in Fig. 15.

The spectral dyadic GF for a two-layered dielectric medium is obtained by applying the spectral domain immittance approach described in [32]. In particular, the infinite array periodic GF for electric source and electric observation point located at the same height $z=z^{\prime}=0$, when the source is a unitary pulse in $\mathbf{r}_{t}$ with unit Fourier transform, is

$$
\overline{\mathbf{G}}^{e}\left(\mathbf{r}_{t} ; \mathbf{r}_{t}^{\prime}\right)=\frac{1}{d_{x} d_{y}} \sum_{i=1}^{\infty} \tilde{\mathbf{G}}^{e}\left(\mathbf{k}_{t_{i}}\right) e^{-j k_{x_{i}} x} e^{-j k_{y_{i}} y}
$$




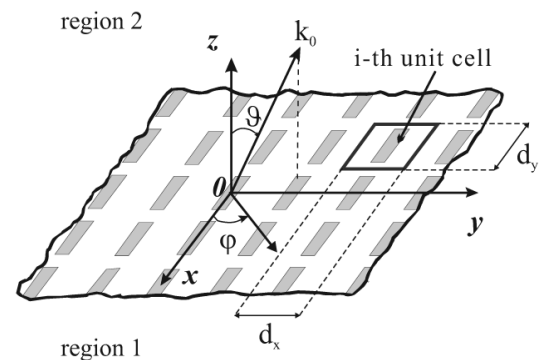

Fig. 14. Infinite periodic dipole array geometry.

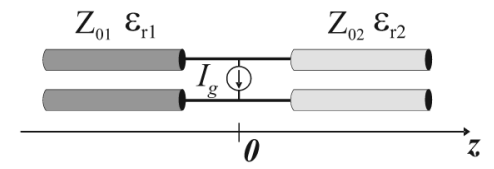

Fig. 15. Equivalent transmission line circuit for a Floquet mode in presence of a dipole array used for the definition of the GF for electric source and electric observation.

where for the sake of simplicity $\overline{\mathbf{G}}^{e}\left(\mathbf{r}_{t} ; \mathbf{r}_{t}^{\prime}\right)=\overline{\mathbf{G}}^{e}\left(\mathbf{r}_{t}, 0 ; \mathbf{r}_{t}^{\prime}, 0\right)$ and $\tilde{\overline{\mathbf{G}}}^{e}\left(\mathbf{k}_{t_{i}}\right)=\tilde{\overline{\mathbf{G}}}^{e}\left(\mathbf{k}_{t_{i}}, 0\right)$. If the current generator $I_{g}$ has unit amplitude, then the expression for the spectral dyadic GF is:

$$
\tilde{\overline{\mathbf{G}}}^{e}\left(\mathbf{k}_{t_{i}}\right)=G_{T M}^{e}\left(\mathbf{k}_{t_{i}}\right) \hat{\mathbf{k}}_{t_{i}} \hat{\mathbf{k}}_{t_{i}}+G_{T E}^{e}\left(\mathbf{k}_{t_{i}}\right) \hat{\boldsymbol{\alpha}} \hat{\boldsymbol{\alpha}}
$$

where

$$
\begin{aligned}
G_{\mathrm{TM}}^{e}\left(\mathbf{k}_{t_{i}}\right) & =-Z_{\mathrm{TM}}\left(\mathbf{k}_{t_{i}}\right) \\
G_{\mathrm{TE}}^{e}\left(\mathbf{k}_{t_{i}}\right) & =-Z_{\mathrm{TE}}\left(\mathbf{k}_{t_{i}}\right) .
\end{aligned}
$$

$Z_{\mathrm{TM}}\left(\mathbf{k}_{t_{i}}\right)=Z_{\mathrm{TM}}\left(\mathbf{k}_{t_{i}}, 0\right)$ and $Z_{\mathrm{TE}}\left(\mathbf{k}_{t_{i}}\right)=Z_{\mathrm{TE}}\left(\mathbf{k}_{t_{i}}, 0\right)$ are the impedances at the section $z=0$ in the equivalent circuit of Fig. 15, and they are equal to the parallel of the input impedances, for the TM and TE $i$ th mode respectively, when looking in each one of the two media.

The expression (29) is defined with respect to the polar basis identified by the unit vectors $\hat{\mathbf{k}}_{t}, \hat{\boldsymbol{\alpha}}$ and $\hat{\mathbf{z}}$

$$
\begin{aligned}
\mathbf{k}_{t_{i}} & =k_{x_{i}} \hat{\mathbf{x}}+k_{y_{i}} \hat{\mathbf{y}} \\
\hat{\mathbf{k}}_{t_{i}} & =\frac{1}{k_{t_{i}}}\left(k_{x_{i}} \hat{\mathbf{x}}+k_{y_{i}} \hat{\mathbf{y}}\right) \\
\hat{\boldsymbol{\alpha}} & =\hat{\mathbf{z}} \times \hat{\mathbf{k}}_{t_{i}}=\frac{1}{k_{t_{i}}}\left(k_{x_{i}} \hat{\mathbf{y}}-k_{y_{i}} \hat{\mathbf{x}}\right) .
\end{aligned}
$$

Introducing the Floquet wave definitions given in (25) and (24) in (28) and (29) leads to

$$
\overline{\mathbf{G}}^{e,(\rho)}\left(\mathbf{r}_{t} ; \mathbf{r}_{t}^{\prime}\right)=-\sum_{i=1}^{\infty}\left(Z^{(\rho)}\left(\mathbf{k}_{t_{i}}\right) \mathbf{e}_{i}^{(\rho)}\left(\mathbf{r}_{t}\right) \mathbf{e}_{i}^{(\rho) *}\left(\mathbf{r}_{t}^{\prime}\right)\right)
$$

where $\rho=\mathrm{TE}$, TM indicates the mode type contribution. At this point, we can use the concept of accessible modes and separate in this expression the contribution of the first $N_{a}$ accessible modes from that of the localized ones

$$
\overline{\mathbf{G}}_{a}^{e,(\rho)}\left(\mathbf{r}_{t}, \mathbf{r}_{t}^{\prime}\right)=-\sum_{i=1}^{N_{a}}\left(Z^{(\rho)}\left(\mathbf{k}_{t_{i}}\right) \mathbf{e}_{i}^{(\rho)}\left(\mathbf{r}_{t}\right) \mathbf{e}_{i}^{(\rho) *}\left(\mathbf{r}_{t}^{\prime}\right)\right)
$$

$$
\begin{aligned}
\overline{\mathbf{G}}^{e,(\rho)}\left(\mathbf{r}_{t}, \mathbf{r}_{t}^{\prime}\right)=-\sum_{i=N_{a}+1}^{\infty}( & Z^{(\rho)} x\left(\mathbf{k}_{t_{i}}\right) \mathbf{e}_{i}^{(\rho)} \\
& \left.\times\left(\mathbf{r}_{t}\right) \mathbf{e}_{i}^{(\rho) *}\left(\mathbf{r}_{t}^{\prime}\right)\right) .
\end{aligned}
$$

The same procedure can be applied for the case of an infinite periodic array of slots cut on a ground plane (magnetic source and magnetic observation point) and leads to the following expression of the infinite periodic GF

$$
\overline{\mathbf{G}}^{h^{(\gamma, \rho)}}\left(\mathbf{r}_{t}, \mathbf{r}_{t}^{\prime}\right)=-\sum_{i=1}^{\infty}\left(Y^{(\gamma, \rho)}\left(\mathbf{k}_{t_{i}}\right) \mathbf{h}_{i}^{(\rho)}\left(\mathbf{r}_{t}\right) \mathbf{h}_{i}^{(\rho) *}\left(\mathbf{r}_{t}^{\prime}\right)\right)
$$

where $Y^{(\gamma, \rho)}=1 /\left(Z^{(\gamma, \rho)}\right)$. In this case $Z^{(\gamma, \rho)}$ are the modal characteristic impedances for the $\rho=\mathrm{TE}, \mathrm{TM}$ modes in regions $\gamma=1,2$.

\section{REFERENCES}

[1] B. A. Munk, Frequency Selective Surfaces: Theory and Design. New York: Wiley, 2000.

[2] S. Monni, G. Gerini, and A. Neto, "Efficient design of a frequency selective surface for a multi functional radar: Theory and measurements," in Proc. Eur. Microwave Conf., Amsterdam, The Netherlands, Oct. 2004.

[3] R. Pous and D. M. Pozar, "A frequency-selective surface using aperture-coupled microstrip patches," IEEE Trans. Antennas Propag., vol. 8, no. 3, 1960.

[4] A. K. Bhattacharyaa, "A numerical model for multilayered microstrip phased-array antennas," IEEE Trans. Antennas Propag., vol. 44, no. 10, pp. 1386-1393, 1996.

[5] T. Itoh, Numerical Techniques for Microwave and Millimeter-Wave Passive Structures. New York: Wiley, 1989.

[6] R. Mittra and S. W. Lee, Analytical Techniques in the Theory of Guided Waves. New York: MacMillan, 1971.

[7] R. Mittra, C. H. Chan, and T. Cwik, "Techniques for analyzing frequency selective surfaces. A review," Proc. IEEE, vol. 76, no. 12, 1988.

[8] M. Guglielmi and A. A. Oliner, "Multimode network description of a planar periodic metal-strip grating at a dielectric interface-Part I: Rigorous network formulations," IEEE Trans. Microw. Theory Tech., vol. 37, no. 3, pp. 534-541, 1989.

[9] R. Orta, R. Tascone, and R. Zich, "A unified formulation for the analysis of general frequency selective surfaces," Electromagn., vol. 5, no. 4, pp. 307-329, 1985.

[10] C. Wan and J. A. Encinar, "Efficient computation of generalized scattering matrix for analyzing multilayered periodic structures," IEEE Trans. Antennas Propag., vol. 43, no. 11, pp. 1233-1242, 1995.

[11] S. Maci, M. Caiazzo, A. Cucini, and M. Casaletti, "A pole-zero matching method for EBG surfaces composed of a dipole FSS printed on grounded dielectric slab," IEEE Trans. Antennas Propag., vol. 53, no. 1 , pp. $70-81,2005$.

[12] I. Palocz and A. A. Oliner, "Equivalent network of multimode planar grating," IEEE Trans. Microw. Theory Tech., vol. 18, no. 5, pp. 244-252, 1970.

[13] G. Gerini, M. Guglielmi, and G. Lastoria, "Efficient integral equation formulations for admittance or impedance representations of planar waveguide junctions," Recent Res. Devel. Microw. Theory Tech., no. 1, pp. 199-206, 1999.

[14] G. Conciauro, M. Guglielmi, and R. Sorrentino, Advanced Modal Analysis. Chichester, U.K.: Wiley, 2000.

[15] G. Gerini and L. Zappelli, "Phased arrays of rectangular apertures on conformal cylindrical surfaces: A multimode equivalent network approach," IEEE Trans. Antennas Propag., vol. 52, no. 7, pp. 1843-1850, 2004.

[16] F. Alessandri, G. Bartolucci, and R. Sorrentino, "Admittance matrix formulation of waveguide discontinuity problems: Computer-aided design of branch guide directional couplers," IEEE Trans. Microw. Theory Tech., vol. 36, no. 2, pp. 394-403, 1988.

[17] S. Edelberg and A. A. Oliner, "Mutual coupling effects in large antenna arrays-Part I: slot arrays," IEEE Trans. Antennas Propag., vol. 8, no. 3, pp. 286-297, 1960. 
[18] T. E. Rozzi and W. F. G. Mecklenbrauker, "Wide-band network modeling of interacting inductive ireses and steps," IEEE Trans. Microwave Theory Tech., vol. 23, pp. 235-244, 1975.

[19] N. Marcuvitz, Waveguide Handbook. New York: McGraw-Hill, 1951.

[20] L. B. Felsen and N. Marcuvitz, Radiation and Scattering of Waves. New York: IEEE Press, 1994, pp. 86-90.

[21] N. Amitay, V. Galindo, and C. P. Wu, Theory and Analysis of Phased Array Antennas. New York: Wiley, 1972.

[22] S. Monni, "Frequency selective surfaces integrated with phased array antennas. analysis and design using multimode equivalent networks," Ph.D. dissertation, Technical University of Eindhoven, Eindhoven, The Netherlands, 2005.

[23] R. F. Harrington, Field Computation by Moment Methods. Malabar, FL: Krieger Publishing, 1968.

[24] V. E. Boria, G. Gerini, and M. Guglielmi, "An efficient inversion technique for banded linear systems," in IEEE Microwave Theory and Tech. Int. Symp. Digest, Boston, MA, Jun. 1997, pp. 1567-1570.

[25] A. K. Bhattacharyaa, "On the convergence of MoM and mode matching solutions for infinite array and waveguide problems," IEEE Trans. Antennas Propag., vol. 51, no. 7, pp. 1599-1606, 2003.

[26] C. Klein and R. Mittra, "Stability of matrix equations arising in electromagnetics," IEEE Trans. Antennas Propag., vol. 21, no. 6, pp. 902-905, 1973.

[27] D. M. Pozar, Microwave Engineering, 2nd ed. New York: Wiley, 1998.

[28] N. Llombart-Juan, "Development of integrated printed array antennas using EBG substrates," Ph.D. dissertation, Departamento de Comunicaciones, Universidad Politecnica de Valencia, Valencia, Spain, 2006.

[29] S. Singh, W. Richards, J. Zinecker, and R. Wilton, "Accelerating the convergence of series representing the free space periodic Green's function," IEEE Trans. Antennas Propag., vol. 38, no. 12, pp. 1958-1962, 1990.

[30] R. Dickie, R. Cahill, H. S. Gamble, V. F. Fusco, A. G. Schuchinsky, and N. Grant, "Spatial demultiplexing in the submillimeter wave band using multilayer free-standing frequency selective surfaces," IEEE Trans. Antennas Propag., vol. 53, no. 6, pp. 1904-1911, 2005.

[31] N. Llombart, A. Neto, and G. Gerini, "Planar circularly symmetric EBG structures for reducing surface waves in printed antennas," IEEE Trans. Antennas Propag., vol. 53, no. 10, pp. 3210-3218, 2005.

[32] T. Itoh, "Spectral domain immitance approach for dispersion characteristics of generalized printed transmission lines," IEEE Trans. Microw. Theory Tech., vol. 28, no. 7, pp. 733-736, 1980.

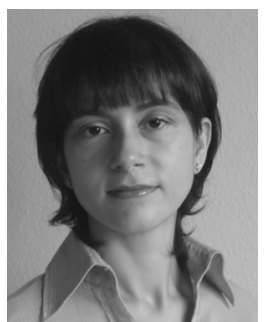

Stefania Monni (S'01-M'06) received the Laurea degree (summa cum laude) in electronic engineering from the University of Cagliari, Italy, in 1999 and the Ph.D. degree in electronic engineering from the Technical University of Eindhoven, The Netherlands, in 2005.

She prepared her Master's thesis at the $\mathrm{Eu}$ ropean Space Research and Technology Centre (ESA-ESTEC) in 1999, where she was employed for one year as a Young Graduate Trainee, working on SAR remote sensing. From 2001 until 2005, she carried out her Ph.D. research at the Netherlands Organization for Applied Scientific Research (TNO), The Hague, The Netherlands, where she is currently employed. Her main research interests concern analysis and design techniques for phased array antennas and frequency selective surfaces.

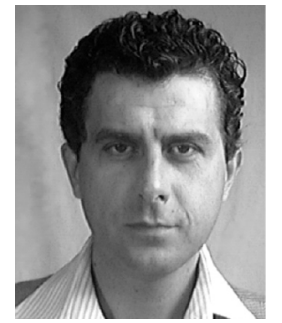

Giampiero Gerini (M'92) received the M.S. degree (summa cum laude) and the Ph.D. in electronic engineering from the University of Ancona, Ancona, Italy, in 1988 and 1992, respectively.

From 1994 to 1997, he was a Research Fellow at the European Space Research and Technology Centre (ESA-ESTEC), Nooordwijk, The Netherlands, where he joined the Radio Frequency System Division. Since 1997, he has been with the Netherlands Organization for Applied Scientific Research (TNO), The Hague, The Netherlands. At TNO Defence, Security and Safety, he is currently Chief Senior Scientist of the Antenna Unit in the Transceivers and Real-time Signal Processing Department. His main research interests are phased array antennas, frequency selective surfaces and integrated front-ends. Since 2007, he is also a part-time Professor at the Technical University of Eindhoven where he holds a Chair on Novel Structures and Concepts for Advanced Antennas.

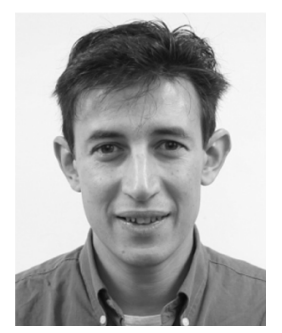

Andrea Neto (M'00) received the Laurea degree (summa cum laude) in electronic engineering from the University of Florence, Italy, in 1994 and the Ph.D. degree in electromagnetics from the University of Siena, Italy, in 2000.

Part of his Ph.D. was developed at the European Space Agency Research and Technology Center, Noordwijk, The Netherlands, where he worked for the antenna section for over two years. In 2000 and 2001, he was a Postdoctoral Researcher at California Institute of Technology, Pasadena, working for the Sub-mm wave Advanced Technology Group. Since 2002, he is a Senior Antenna Scientist at TNO Defence, Security and Safety, Den Haag, The Netherlands.

His research interests are in the analysis and design of antennas, with emphasis on arrays, dielectric lens antennas, wide band antennas and EBG structures.

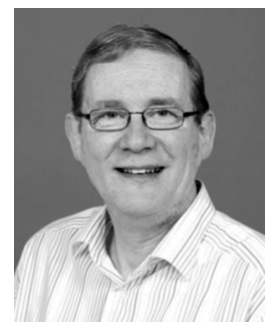

Anthon G. Tijhuis (M'88) was born in Oosterhout N.B., The Netherlands, in 1952 . He received the M.Sc. degree in theoretical physics from Utrecht University, Utrecht, The Netherlands, in 1976, and the Ph.D. degree (cum laude) from the Delft University of Technology, Delft, The Netherlands, in 1987.

From 1976 to 1986 and 1986 to 1993 , he was an Assistant and Associate Professor with the Laboratory of Electromagnetic Research, Faculty of Electrical Engineering, Delft University of Technology. In 1993, he became a Full Professor of electromagnetics with the Faculty of Electrical Engineering, Eindhoven University of Technology, Eindhoven, The Netherlands. He has been a Visiting Scientist with the University of Colorado at Boulder, the University of Granada, Granada, Spain, the University of Tel Aviv, Tel Aviv, Israel, and with McDonnell Douglas Research Laboratories, St. Louis, MO. Since 1996, he has been a Consultant with TNO Defence, Security, and Safety, The Hague, The Netherlands. His research interests are the analytical, numerical, and physical aspects of the theory of electromagnetic waves. In particular, he is involved with efficient techniques for the computational modeling of electromagnetic fields and their application to detection and synthesis problems from several areas of electrical engineering. 